\title{
Dynamics of Type-3 Wind Turbine Generator Models
}

\author{
Ian A. Hiskens \\ University of Michigan
}

\begin{abstract}
The influence of wind turbine generators (WTGs) on power system dynamic performance is becoming increasingly important as wind generation grows. The dynamic behaviour of WTGs should therefore be thoroughly understood. The report analyzes dynamic models of type-3 WTGs, and in particular the WECC generic model. The behaviour of such models is governed by interactions between the continuous dynamics of state variables, and discrete events associated with limits. It is shown that these interactions can be quite complex, and may lead to switching deadlock that prevents continuation of the trajectory. Switching hysteresis is proposed for eliminating deadlock situations. Various type-3 WTG models include control blocks that duplicate integrators. It is shown that this leads to non-uniqueness in the conditions governing steady-state, and may result in pre- and post-disturbance equilibria not coinciding. It also gives rise to a zero eigenvalue in the linearized WTG model.
\end{abstract}

\section{Introduction}

The dynamic behaviour of wind turbine generators (WTGs) is quite different to that of synchronous generators. It is to be expected, therefore, that the dynamic performance of power systems may change as traditional generation is displaced by ever increasing numbers of WTGs. Numerous studies have investigated this issue, and have drawn various conclusions $[1,2,3,4,5]$. This current report does not address the system-wide implications of large-scale wind generation per se. Rather, it focuses on the dynamic modelling of WTGs. In particular, the modelling of type-3 WTGs is considered, as they are currently the dominant technology for new wind farm developments. Such WTGs are also known as doubly fed induction generators (DFIGs) or doubly fed asynchronous generators. A schematic is provided in Figure 1.

The accuracy of system studies depends on the fidelity of the underlying models $[6,7]$. Accordingly, the modelling of type- 3 WTGs has received considerable attention, see $[1,8$, $9,10,11,12]$ and references therein. Turbine manufacturers routinely develop and maintain accurate models for their products, though disclosure of those models is highly restricted. In some cases they have released models that describe functionally similar behaviour [13], though such practise is not common. Regional reliability organizations need to exchange models and data that are relevant to their jurisdiction. This has motivated the development of generic models that can be used to capture the functional characteristics of a wide variety of type-3 WTGs $[14,15]$.

As indicated in Figure 1, the electrical characteristics of type-3 WTGs are governed by interactions between the wound-rotor induction machine and the back-to-back inverter. The inverter excites the rotor of the induction machine with a variable $\mathrm{AC}$ source. This provides control of the rotor flux frequency, enabling the rotor shaft frequency to optimally track 


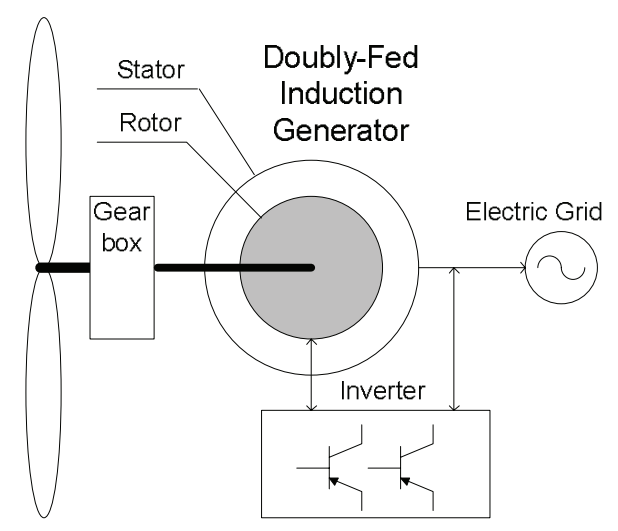

Figure 1: Schematic diagram of a type-3 wind turbine generator.

wind speed [16]. The inverter response time is very fast relative to electromechanical time constants. As a result, the natural dynamics of the induction machine are largely masked from the power system. The dynamic behaviour of a type-3 WTG, as seen from the grid, is therefore dominated by controller response rather than physical characteristics. This is in marked contrast to traditional synchronous generators, where behaviour is governed by device physics.

Controller limits play an integral role in the dynamic performance of type-3 WTGs, with further details provided in Section 2. Intrinsic interactions between continuous dynamics and limit-induced discrete events suggest that type-3 WTGs may be classified as hybrid dynamical systems $[17,18,19]$. It will be shown in Section 3 that the resulting hybrid dynamics may, in fact, lead to unusual forms of behaviour. The hybrid nature of dynamics also has implications for small disturbance studies.

The studies presented in this report focus on the WECC generic type-3 model [15]. This model has been chosen because it is widely used, and is indicative of type- 3 models that are generally available. All such generic models are an approximation of the actual dynamics exhibited by a WTG. It is important, though, that this approximation reflects the physical reality of the modelled device.

The report is organized as follows. Section 2 provides a thorough description of the WECC type-3 WTG model. It is shown in Section 3 that non-windup limiter models may cause switching deadlock, preventing trajectory continuation in the usual sense. Alternative model formulations that circumvent such behaviour are discussed. Section 4 shows that the model allows multiple equilibria, and discusses the implications. Small disturbance analysis is considered in Section 5, and conclusions are presented in Section 6.

\section{Type-3 WTG model}

The WECC type-3 wind turbine generator model is defined in [15]. The complete WTG model is divided into four functional blocks, as indicated in Figure 2. This report is primarily concerned with the dynamic interactions of the converter control model WT3E, the pitch control model WT3P, and the wind turbine model WT3T. Accordingly, only those models are described in detail in the following analysis. 


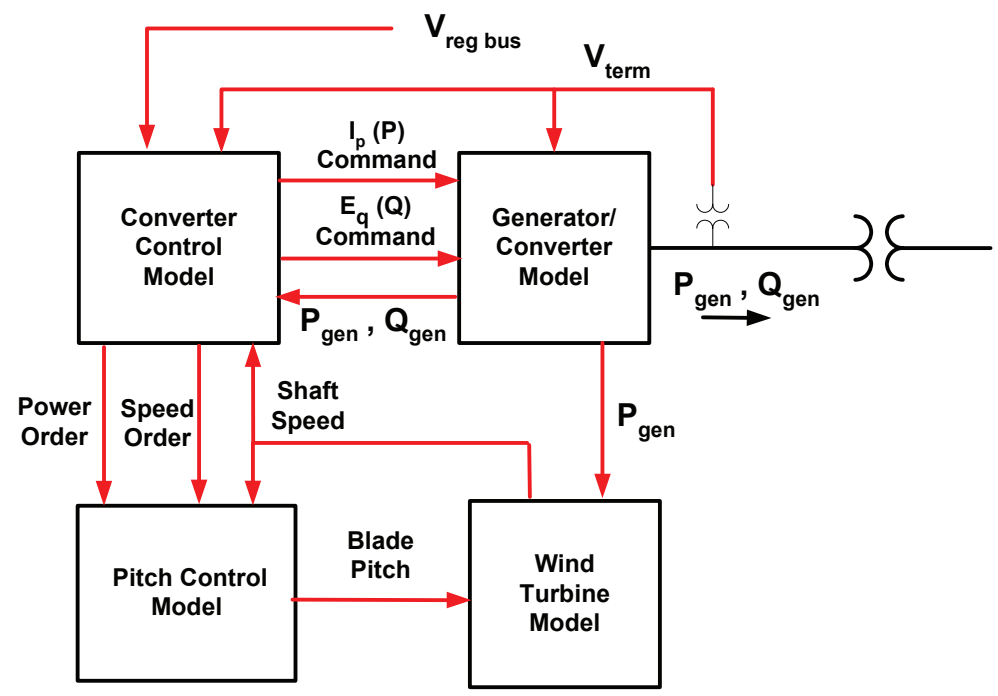

Figure 2: Type-3 WTG dynamic model connectivity, from [15].

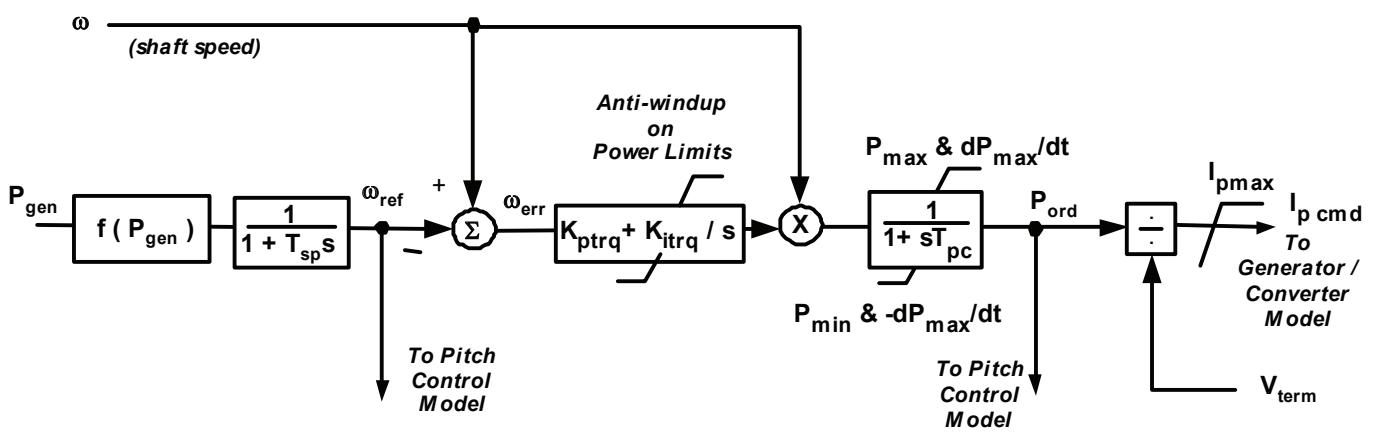

Figure 3: Converter control model WT3E, from [15].

\subsection{Converter control model WT3E}

The converter control model is composed of separate active and reactive power control functions. Reactive power control is very fast, due to the power electronic converter. This report focuses on the slower dynamics associated with interactions between active power (torque) control, pitch control, and the coupling through the shaft dynamics. Accordingly, only the active power model, which is shown in Figure 3, will be discussed. Again, full details of the reactive power controller are provided in [15].

The non-windup (anti-windup) limits on the PI block in the centre of Figure 3 are driven by the non-windup $P_{\max } / P_{\min }$ limits associated with the $P_{\text {ord }}$ lag block. The model documentation stipulates that:

(i) If $P_{\text {ord }}$ is on its $P_{\max }$ limit and $\omega_{\text {err }}$ (the input to the PI block) is positive, then the $K_{i t r q}$-integrator is blocked, i.e., the state $T_{\omega}$ of that integrator is frozen.

(ii) If $P_{\text {ord }}$ is on the $P_{\text {min }}$ limit and $\omega_{e r r}$ is negative, then the $K_{i t r q}$-integrator state is frozen. This form of non-windup limit is unusual, though a precedence can be found in Annex E.5 


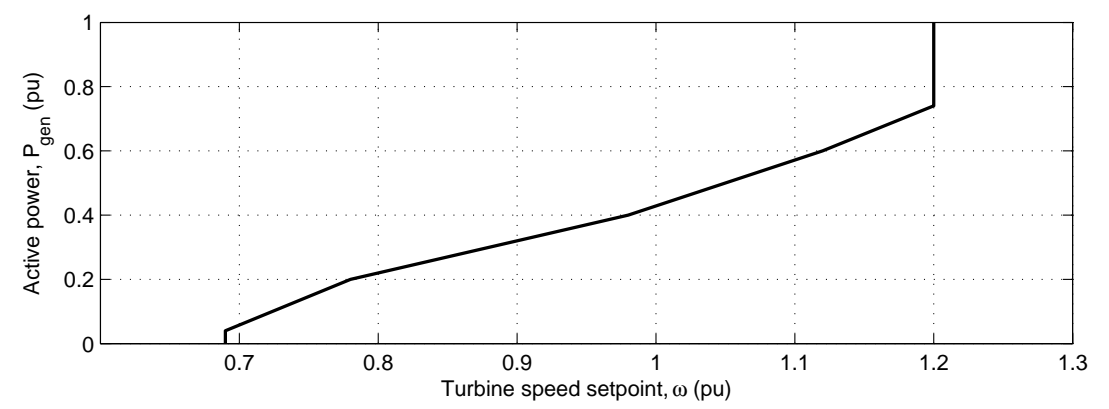

Figure 4: Speed-power relationship, $f\left(P_{\text {gen }}\right)$.

of IEEE Standard 421.5-2005 [20]. It will be shown in Section 3 that such non-windup logic can result in switching deadlock [21].

The function $f\left(P_{g e n}\right)$ is typically modelled as a piece-wise affine function. WECC default parameters, which are provided in Appendix C, produce the curve shown in Figure 4.

Assembling all the equations for the model gives,

$$
\begin{aligned}
\frac{d \omega_{\text {ref }}}{d t} & =\frac{1}{T_{s p}}\left(f\left(P_{\text {gen }}\right)-\omega_{\text {ref }}\right) \\
\frac{d T_{\omega}}{d t} & =K_{\text {itrq }}\left(\omega-\omega_{\text {ref }}\right) \times y_{\text {freeze }} \\
\frac{d P_{\text {ord }}}{d t} & =P_{\text {ord }, r t l m} \times y_{m x, s w} \times y_{m n, s w} \\
P_{\text {ord }, \text { rate }} & =\frac{1}{T_{p c}}\left(\omega\left(T_{\omega}+K_{\text {ptrq }}\left(\omega-\omega_{\text {ref }}\right)\right)-P_{\text {ord }}\right)
\end{aligned}
$$

together with the switched equations (27)-(30) that are given in Appendix A.

The value of the model (1)-(4) and (27)-(30) is that it provides a precise, unambiguous description of dynamic behaviour. This level of detail is vitally important for analyzing the model idiosyncracies that are discussed in later sections.

\section{$2.2 \quad$ Pitch control model WT3P}

The pitch control model WT3P is shown in Figure 5. Of particular interest is the implementation of the non-windup limiter on the pitch angle $\theta$. As stated in the model documentation,

"The Pitch Control and Pitch Compensation integrators are non-windup integrators as a function of the pitch, i.e., the inputs of these integrators are set to zero when the pitch is in limits $\left(P I_{\max }\right.$ or $\left.P I_{\min }\right)$ and the integrator input tends to force the pitch command further against its limit."

To illustrate, consider the case where $\theta$ is on its lower limit $P I_{\min }$. A negative input to the pitch-control integrator would cause the corresponding state $x_{p}$ to reduce, which in turn would force $\theta$ further against its $P I_{\min }$ limit. To prevent that wind-up effect, the integrator is blocked under such conditions. Similarly, the pitch-compensation integrator is blocked when its input is negative. When $\theta$ is on its upper limit $P I_{\max }$, blocking of the up-stream integrators occurs when their respective inputs are positive. 


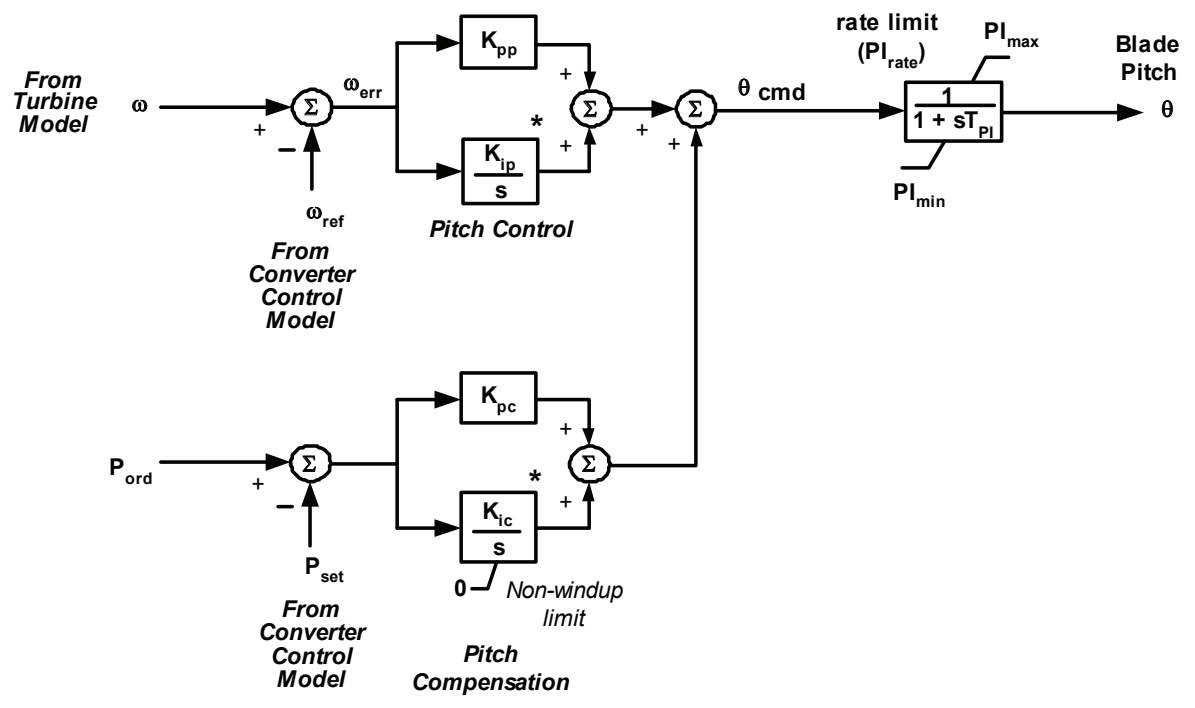

Figure 5: Pitch control model WT3P, from [15].

This blocking philosophy is the same as that employed in the converter control model WT3E, as discussed in Section 2.1. It should again be mentioned that such blocking can result in switching deadlock. This will be explored in Section 3.

The equations describing the WT3P model can be written,

$$
\begin{aligned}
\frac{d x_{p}}{d t} & =K_{i p}\left(\omega-\omega_{r e f}\right) \times y_{f r, 1} \\
\frac{d x_{c}}{d t} & =K_{i c}\left(P_{o r d}-P_{s e t}\right) \times y_{f r, 2} \times y_{s w} \\
\frac{d \theta}{d t} & =\theta_{r t l m} \times y_{m x, s w} \times y_{m n, s w} \\
\theta_{\text {rate }} & =\frac{1}{T_{P I}}\left(\theta_{c m d}-\theta\right) \\
\theta_{c m d} & =x_{p}+x_{c}+K_{p p}\left(\omega-\omega_{r e f}\right)+K_{p c}\left(P_{\text {ord }}-P_{\text {set }}\right)
\end{aligned}
$$

along with the switched equations (31)-(36) that are provided in Appendix B.

\subsection{Wind turbine model WT3T}

The single-mass wind turbine model WT3T from [15] is shown in Figure 6. A two-mass model is also provided in [15], but the single-mass model suffices for the discussions in this report. The model consists of two parts, 1) a simplified model of the aerodynamic relationship between blade pitch $\theta$ and mechanical power $P_{\text {mech }}$, and 2) a model of the shaft dynamics. The damping constant $D$ is always zero, so the single-mass WT3T model can be described by,

$$
\begin{aligned}
\frac{d \omega}{d t} & =\frac{1}{2 H \omega}\left(P_{\text {mech }}-P_{g e n}\right) \\
P_{\text {mech }} & =P_{\text {mo }}-K_{\text {aero }} \theta\left(\theta-\theta_{o}\right) .
\end{aligned}
$$




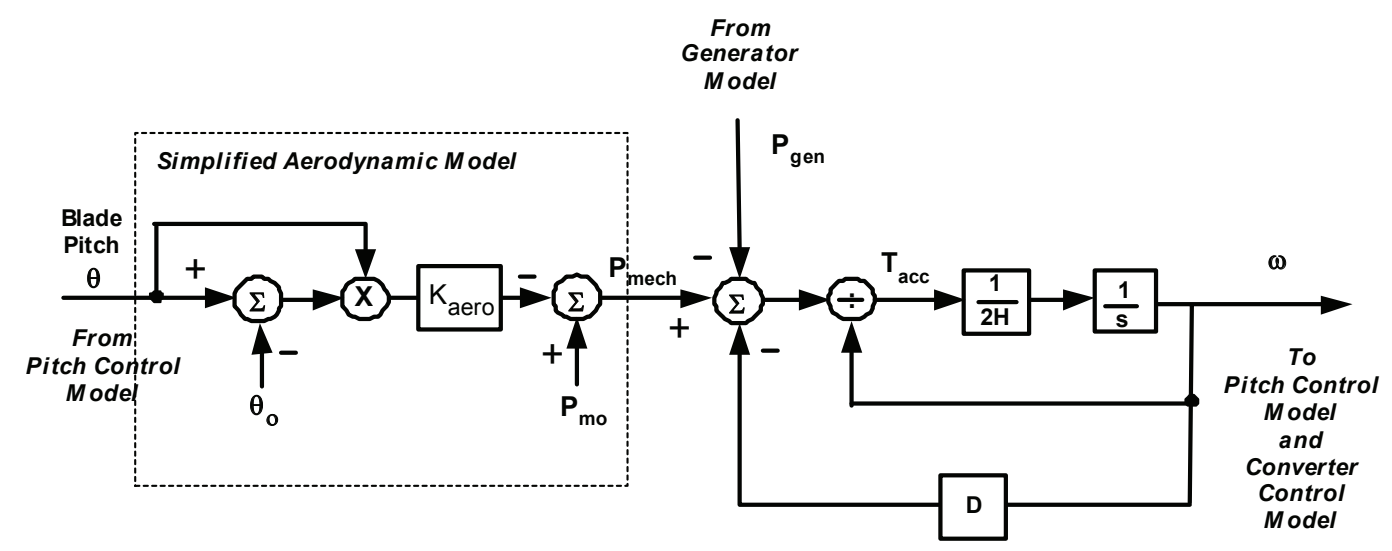

Figure 6: Single-mass turbine model WT3T, from [15].

\subsection{Hybrid system model}

It is clear from (1)-(11) and (27)-(36) that the WTG model is composed of:

i) Differential and algebraic equations that describe continuous behaviour of the associated states, and

ii) Discrete events that introduce nonsmooth behaviour through switching actions.

Models that involve such interactions between continuous dynamics and discrete events have become known as hybrid dynamical systems [17, 18, 19]. The discrete events introduce rich forms of behaviour that are not exhibited by smooth systems described by differential (and possibly coupled algebraic) equations. In fact, it will be shown in Section 3 that both the WT3E and WT3P models are susceptible to switching deadlock, where a discrete state should simultaneously take two different values. This situation is impossible, of course; the trajectory is not defined (in the usual sense) beyond such an impasse.

The WTG model (1)-(11), (27)-(36) has been formulated according to the differentialalgebraic impulsive switched (DAIS) structure described in [19, 22]. A subtle (but technically important) modification was required however. In the original DAIS definition, switched algebraic equations had the form

$$
0= \begin{cases}g^{+}(x, y), & y_{s}>0 \\ g^{-}(x, y), & y_{s}<0\end{cases}
$$

where behaviour is undefined if the trigger variable $y_{s}$ remains at zero. In the case of a WTG though, it is quite common for the pitch angle $\theta$ to be initialized on its lower limit $P I_{\text {min }}$. To cater for that (and similar) situations, the DAIS definition has been altered to allow the trigger variable $y_{s}$ to remain at zero, giving the slightly modified switching description,

$$
0= \begin{cases}g^{+}(x, y), & y_{s} \geq 0 \\ g^{-}(x, y), & y_{s}<0\end{cases}
$$

In the case of the non-windup limits within the WTG model, (28)-(29) and (32)-(34), it has been arbitrarily decided that the integrator should remain active when its state lies on 
the limit, i.e., when the trigger variable $y_{s}=0$. The integrator only becomes blocked when the input seeks to force the state across the limit, resulting in $y_{s}<0$.

Technical issues arise when an equilibrium point coincides with a switching condition $y_{s}=0$. If the equilibrium is asymptotically stable, then generically, as the trajectory approaches the equilibrium point, the time between subsequent switching events will progressively diminish. In the limit, switching will (theoretically) become infinitely fast. Also, linearization about the equilibrium point is not defined, as the vector field is not smooth. Consequently, small disturbance analysis is not possible. This latter point is explored further in Section 5.

\section{Trajectory deadlock}

\subsection{Background}

Conceptually, deadlock refers to the situation where a trajectory encounters a condition that precludes further progress. Such behaviour is unusual for systems described by continuous dynamics $^{1}$, though differential-algebraic systems can experience deadlock in the form of algebraic singularity ${ }^{2}[24,25]$.

Hybrid dynamical systems, on the other hand, are more prone to deadlock, due to their inherent interactions between continuous dynamics and discrete events. In this context, deadlock has been formally defined in [21]. The form of deadlock of particular relevance to WTG modelling is known as chattering Zeno, which refers to situations where "the discrete component infinitely jumps instantaneously between different domains, while the continuous component remains unchanged" [21]. Subsequent sections describe this behaviour in the context of the type-3 WTG model, and provide an alternative model formulation that alleviates the deadlock phenomenon.

It should be noted that because deadlock precludes continuation of a trajectory, numerical simulation techniques that accurately capture hybrid system dynamics cannot proceed beyond the deadlock point. Conversely, simulators that continue through deadlock cannot be truly implementing the hybrid system model. Special techniques have been developed for continuing approximate solutions beyond deadlock, with Filippov solution concepts forming the basis for those methods [26]. Such concepts are required, for example, in analyzing sliding mode behaviour [27]. They are not pursued in this report.

\subsection{Deadlock in WTG models}

The switching logic that gives rise to trajectory deadlock in the type-3 WTG model can be explained with the aid of the simplified model of Figure 7. Referring to Figure 5, it can be seen that this reduced model is equivalent to the output lag block and one of the upstream PI regulators of the WT3P model.

Consider the case where $x_{1}$ is on its lower limit $x_{m i n}$, and the input $u$ is negative. According to the non-windup logic of WT3P, the $x_{2}$-integrator would be blocked. Assume that $u$ is increasing, though remains negative. This increase in $u$ will translate directly into an increase in $y$, as $x_{2}$ is constant. With increasing $y$, conditions conducive to deadlock occur when $y$ reaches $x_{1}=x_{\text {min }}$. This may be explained by noting that $\dot{x}_{1}=\left(y-x_{1}\right) / T$, and hence

\footnotetext{
${ }^{1}$ Technically, deadlock cannot occur if the vector field is Lipschitz [23].

${ }^{2}$ In this case, the term impasse is often used rather than deadlock.
} 


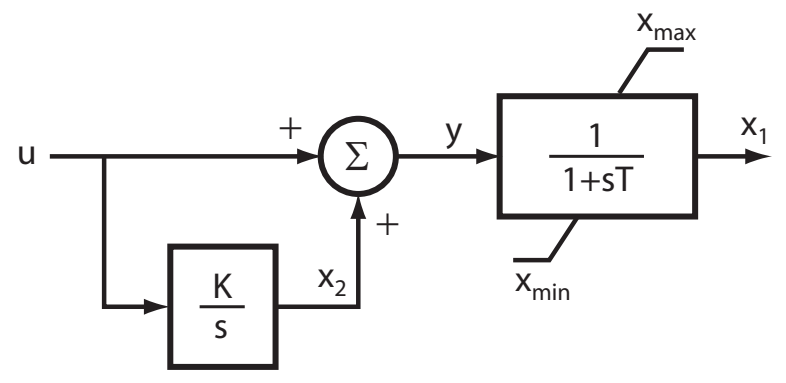

Figure 7: Simplified representation of non-windup limited block and upstream PI controller.

$\dot{x}_{1}=0$ at the point where $y$ encounters $x_{1}=x_{\min }$. The evolution of $x_{1}$ from that point is therefore governed by $\ddot{x}_{1}$, which can be written

$$
\ddot{x}_{1}=\frac{1}{T}\left(\dot{y}-\dot{x}_{1}\right)=\frac{1}{T} \dot{y}=\frac{1}{T}\left(\dot{u}+\dot{x}_{2}\right) .
$$

If the $x_{2}$-integrator is blocked, $\dot{x}_{2}=0$, giving $\ddot{x}_{1}=\frac{1}{T} \dot{u}>0$ because $u$ is increasing. In this case, $x_{1}$ will tend to increase away from the $x_{\min }$ limit, and the $x_{2}$-integrator will become unblocked. On the other hand, if the $x_{2}$-integrator is unblocked, the sign of $\ddot{x}_{1}$ is given by $\dot{u}+\dot{x}_{2}=\dot{u}+K u$, which may be negative. If so, $x_{1}$ will tend to decrease onto the $x_{\text {min }}$ limit, blocking the $x_{2}$-integrator. A contradiction arises: if the $x_{2}$-integrator is blocked it should unblock, but if it's unblocked it should block.

Returning to the type-3 WTG model, this deadlock phenomenon can be illustrated using the WECC test system and default parameters that are provided in Appendix C. Resulting trajectories are shown in Figure 8 . For the sake of clarity, only the pitch-compensation integrator will be discussed, though the pitch-control integrator exhibits similar behaviour. It should be noted that in order to generate the trajectories shown in Figure 8, it was necessary to introduce hysteresis into the switching process associated with the pitch angle non-windup limit. The implementation of hysteresis is discussed in Section 3.3.

The pitch angle $\theta$ is initially in steady-state on the lower non-windup limit, where $\theta_{0}=$ $P I_{\min }=0$ deg. In response to the disturbance, $\theta$ undergoes a transient increase, before returning to $P I_{\text {min }}$ at $5.6 \mathrm{sec}$. The error signal $x_{c, e r r}=P_{\text {ord }}-P_{\text {set }}$ driving the pitch-compensation integrator is negative at that time, so the corresponding state $x_{c}$ is frozen. Over the subsequent period, $\theta$ and $x_{c}$ remain frozen, but the signal $\theta_{e r r}=\theta_{c m d}-\theta$, which drives variations in $\theta$, steadily increases until reaching zero ${ }^{3}$ at around $7 \mathrm{sec}$. At that point, $\theta$ should transition from blocked to unblocked, so the pitch-compensation integrator driving $x_{c}$ should also unblock. But notice that $x_{c, e r r}$ is negative, so as soon as the integrator unblocks, $x_{c}$ will decrease, driving $\theta_{\text {err }}$ negative. This forces $\theta$ back onto its $P I_{\min }$ limit, blocking $x_{c}$ again. But with $x_{c}$ blocked, $\theta_{\text {err }}$ increases above zero, and $x_{c}$ is unblocked. Without hysteresis, this process would repeat ad infinitum.

In summary, at the point where $\theta_{\text {err }}$ encounters zero,

- If $\theta$ is blocked, then $\theta$ and $x_{c}$ should unblock.

- If $\theta$ is unblocked, then $\theta$ and $x_{c}$ should block.

\footnotetext{
${ }^{3}$ The hysteresis implementation actually allows $\theta_{\text {err }}$ to rise a little beyond zero before the $x_{c}$-integrator is unblocked. Further details are provided in Section 3.3.
} 


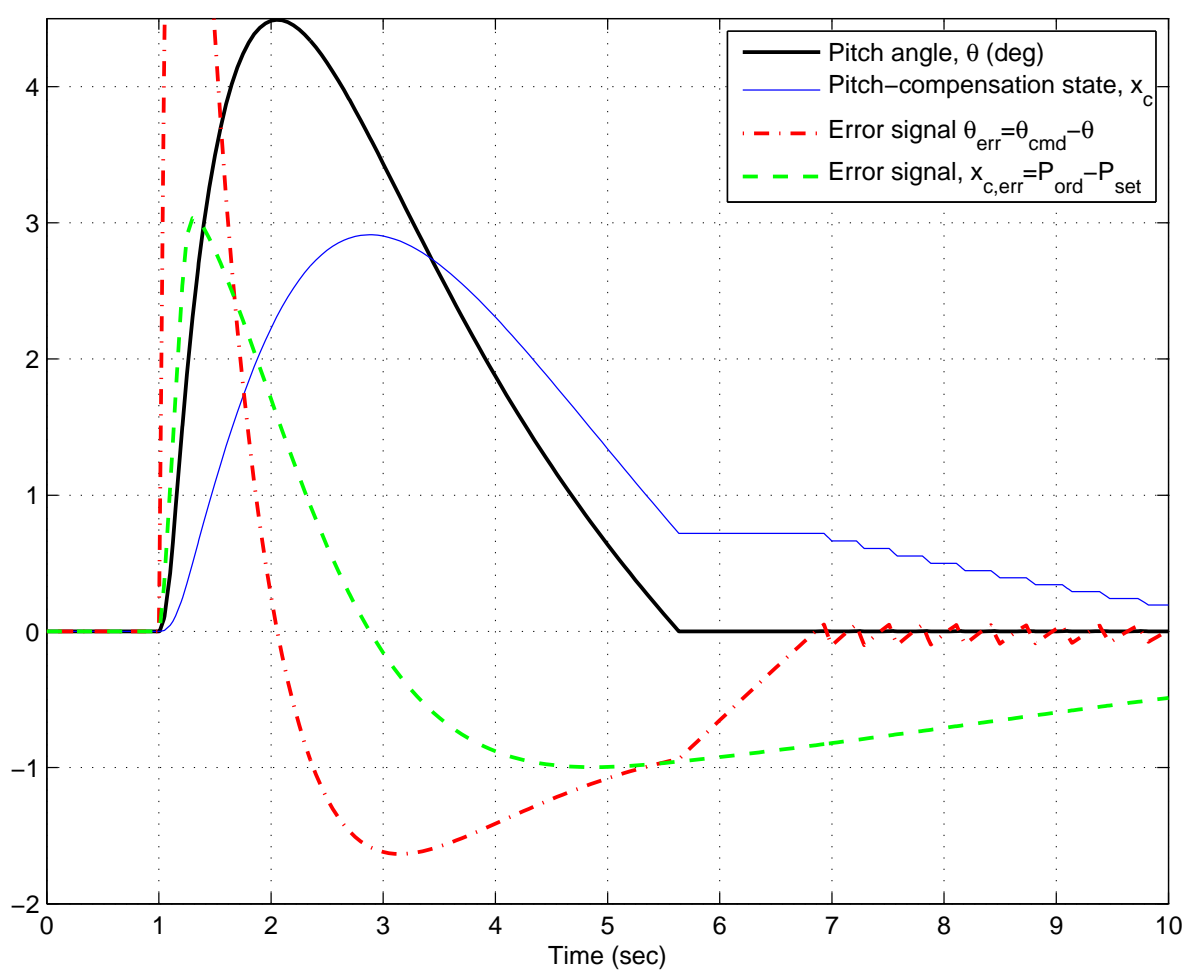

Figure 8: Large-disturbance response of pitch states.

In other words, at the instant when $\theta_{\text {err }}=0$, the discrete state describing integrator blocking undergoes infinitely many switches, preventing the continuation of the trajectory.

This impasse can be overcome by implementing hysteresis in the blocking/unblocking process, as described in the following section.

\subsection{Implementation of hysteresis}

The explanation of hysteresis will refer to Figure 9. This is an expanded view of the relevant time interval of Figure 8. In order to provide a clearer view of behaviour, however, the hysteresis band has been widened from 0.002 in Figure 8 to 0.05 in Figure 9.

At $6.85 \mathrm{sec}$, the error signal $\theta_{\text {err }}$ crosses through zero. Upon doing so, the integrator driving the pitch angle $\theta$ is unblocked, so $\theta$ begins to increase. At $7.3 \mathrm{sec}, \theta$ encounters the hysteresis threshold, whereupon the pitch-compensation integrator is unblocked. The error signal $x_{c, e r r}$ driving that integrator is negative, as shown in Figure 8, so $x_{c}$ immediately begins to reduce. This causes $\theta_{\text {err }}$ to reduce. Eventually $\theta_{\text {err }}$ goes negative, and $\theta$ begins to fall, encountering the non-windup limit $P I_{\min }=0$ at $7.6 \mathrm{sec}$. When that limit is encountered, $\theta$ and $x_{c}$ are immediately blocked, so $\theta_{\text {err }}$ again begins to increase. The process then repeats.

The actual implementation of the hysteresis logic is presented in [28]. 


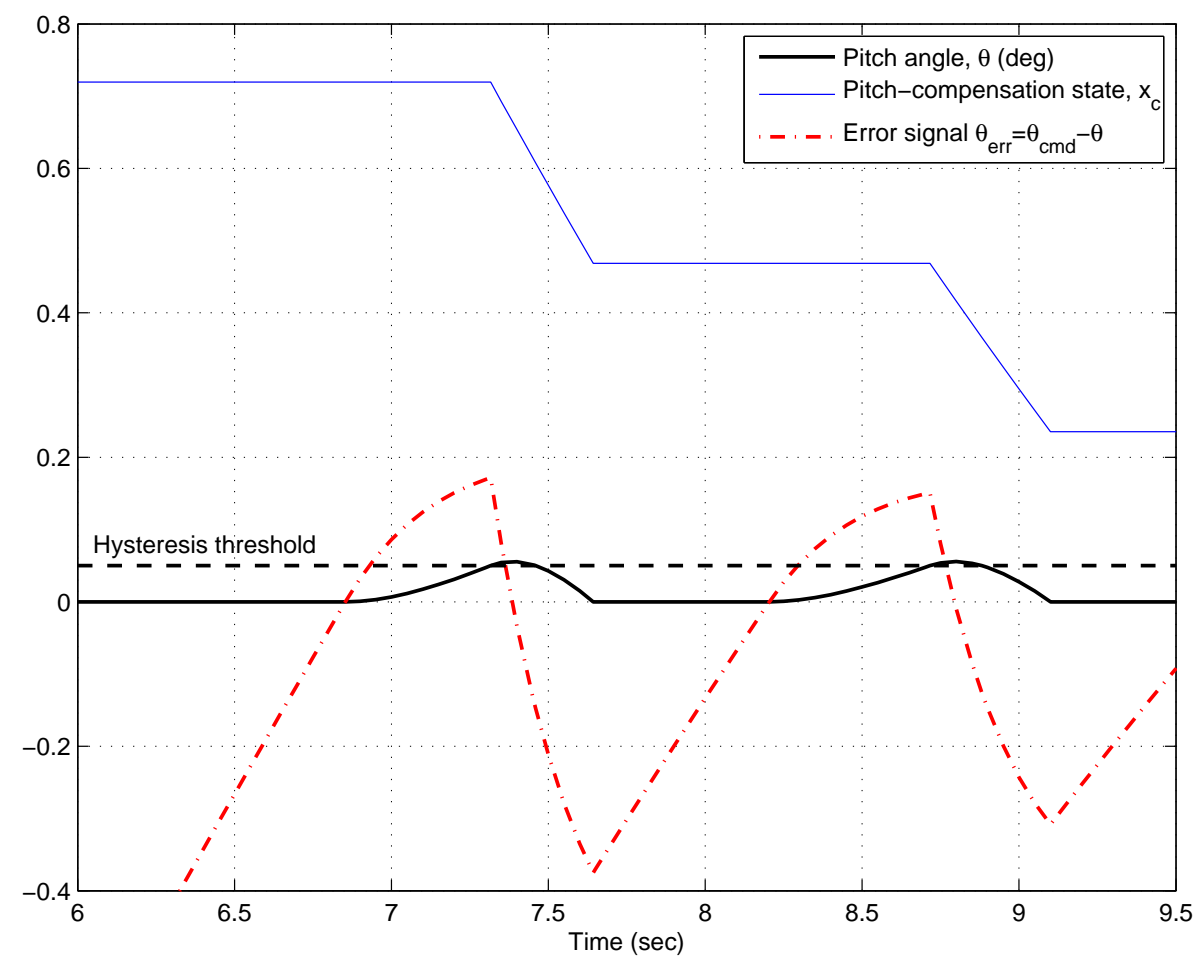

Figure 9: Expanded view of hysteresis behaviour.

\section{Equilibrium conditions}

\subsection{Initialization}

For the initial point to be in equilibrium, the derivatives in (1)-(3), (5)-(7) and (10) must be set to zero. Notice though that because (2) and (5) are effectively duplicate integrators, they both contribute exactly the same equilibrium equation, $\omega-\omega_{\text {ref }}=0$. The redundant equation will be ignored when assembling the complete set of initialization equations.

The status of the switched equations must also be consistent with equilibrium conditions. It is safe to assume that none of the limits in the converter control model WT3E would be active during normal steady-state operation. Under that assumption, $P_{\text {ord }}$ will equal the electrical power $P_{\text {gen }}$ delivered to the WTG terminal bus. This relationship will be used to eliminate $P_{g e n}$ from the equilibrium equations.

In the case of the pitch control model WT3P, limits may be active at steady-state. For wind conditions up to rated wind-speed, the pitch angle $\theta$ would normally sit at its minimum limit $P I_{\text {min }}$. Blocking the associated integrator would, however, leave the initial value of $\theta_{c m d}$ undefined. The initialization process must therefore override integrator blocking, or equivalently, assume $\theta$ lies infinitesimally above the $P I_{\min }$ limit.

The pitch compensation non-windup limiter of WT3P has the equilibrium characteristic shown in Figure 10. If the input $P_{\text {ord }}-P_{\text {set }}<0$ at steady-state, then $x_{c}$ will be forced to its lower limit of zero. On the other hand, if $P_{\text {ord }}-P_{\text {set }}=0$ at steady-state, then $x_{c}$ may take any non-negative value. Summarizing this relationship gives,

$$
x_{c} \geq 0, \quad P_{\text {set }}-P_{\text {ord }} \geq 0, \quad x_{c}\left(P_{\text {set }}-P_{\text {ord }}\right)=0,
$$




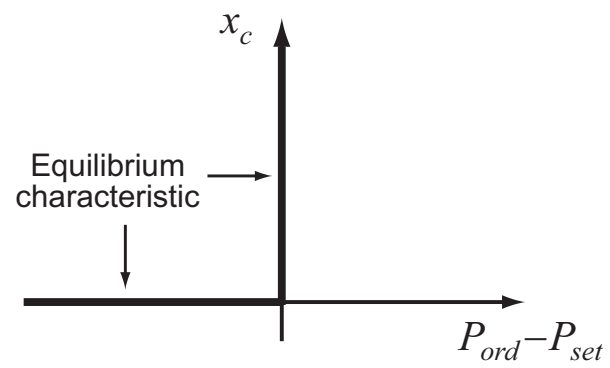

Figure 10: Equilibrium characteristic for the pitch compensation integrator.

which is a complementarity condition $[29,30]$ that can be expressed compactly using standard notation,

$$
0 \leq x_{c} \perp\left(P_{\text {set }}-P_{\text {ord }}\right) \geq 0 .
$$

The resulting initialization equations can be written,

$$
\begin{aligned}
& 0=f\left(P_{\text {ord }}\right)-\omega_{\text {ref }} \\
& 0=\omega-\omega_{\text {ref }} \\
& 0=\omega T_{\omega}-P_{\text {ord }} \\
& 0=P_{\text {mo }}-K_{\text {aero }} \theta\left(\theta-\theta_{o}\right)-P_{\text {mech }} \\
& 0=P_{\text {mech }}-P_{\text {ord }} \\
& 0=x_{p}+x_{c}+K_{\text {pc }}\left(P_{\text {ord }}-P_{\text {set }}\right)-\theta_{\text {cmd }} \\
& 0=\theta_{\text {cmd }}-\theta
\end{aligned}
$$

together with (12). The dependent state variables are $\omega_{r e f}, T_{\omega}, P_{\text {ord }}, x_{p}, x_{c}, \theta, \omega, \theta_{c m d}$ and $P_{\text {mech }}$, while $K_{\text {aero }}, K_{p c}, P_{m o}$ and $P_{\text {set }}$ are parameters. By definition, $\theta_{o}$ is the specified initial value for the pitch angle $\theta$, thereby providing a further initialization equation,

$$
\theta-\theta_{o}=0 .
$$

Because $P_{m o}$ and $P_{\text {set }}$ are independent parameters, it is important to consider initialization for the three cases, $P_{m o}<P_{\text {set }}, P_{m o}=P_{\text {set }}$ and $P_{m o}>P_{\text {set }}$. To do so, first notice that (16)-(17) and (20) together infer $P_{m o}=P_{\text {ord }}$ at initialization. Therefore, for the case $P_{m o}=$ $P_{\text {ord }}<P_{\text {set }}$, the complementarity condition (12), expressed visually in Figure 10, ensures that $x_{c}=0$. In the second case, when $P_{m o}=P_{\text {ord }}=P_{\text {set }}$, (12) only specifies that $x_{c} \geq 0$. It follows that the initialization description consists of only eight independent equations describing nine variables. The set of equations is under-determined. This can be confirmed by noting that $x_{c}$ and $x_{p}$ appear only in (18), and therefore cannot be uniquely determined. To resolve this situation, (12) should be replaced at initialization by an equation that assigns a specific initial value to $x_{c}$ or $x_{p}$. For consistency with the $P_{m o}<P_{\text {set }}$ case, it is convenient to replace (12) by

$$
x_{c}=0 .
$$

The third case $P_{m o}=P_{\text {ord }}>P_{\text {set }}$ implies the integrator would be driven by a sustained positive input. The WT3P model does not define an upper limit though, so equilibrium could not be achieved. 


\subsection{Post-disturbance steady-state}

For well-posed dynamical models, the post-disturbance steady-state should match the predisturbance (initial) equilibrium when pre- and post-disturbance parameter sets are identical. This is generically not the case for the WECC type-3 WTG model. The following analysis shows that this unusual behaviour is due to switching associated with the duplicate integrators (2) and (5).

The initialization equations (12)-(19) govern post-disturbance steady-state conditions, as they were obtained by setting derivatives to zero. Initialization also made use of the auxiliary equation (20), but that equation plays no role as the system evolves towards steady-state. As a consequence of discarding (20), the description of steady-state conditions is under-determined.

This indeterminacy is resolved when the duplicate integrators (2) and (5) remain unblocked for the entire time horizon. In that case, the integrator states can be written in integral form,

$$
\begin{aligned}
& T_{\omega}(t)=T_{\omega}^{o}+K_{\text {itrq }} \int_{0}^{t}\left(\omega(\tau)-\omega_{\text {ref }}(\tau)\right) d \tau \\
& x_{p}(t)=x_{p}^{o}+K_{i p} \int_{0}^{t}\left(\omega(\tau)-\omega_{r e f}(\tau)\right) d \tau
\end{aligned}
$$

where $T_{\omega}^{o}$ and $x_{p}^{o}$ are the initial values for the respective states. Equating the integrals in (22) and (23) gives the affine relationship,

$$
x_{p}(t)=\frac{K_{i p}}{K_{i t r q}} T_{w}(t)+\left(x_{p}^{0}-\frac{K_{i p} T_{w}^{0}}{K_{i t r q}}\right)
$$

which implies that any variation in $T_{\omega}(t)$ will be matched by a corresponding variation in $x_{p}(t)$. This relationship provides the extra equation required to uniquely determine the postdisturbance steady-state, and in fact implies that if all parameters remain unchanged, the system will evolve to a steady-state that exactly matches the initialization point.

The assumption that the duplicate integrators remain unblocked for all time is seldom true, however. Blocking of one or other of the integrators will alter the corresponding integral term in (22) or (23), invalidating the relationship (24). Under such conditions, it becomes impossible for both $T_{\omega}$ and $x_{p}$ to evolve back to their initial values. Consequently, the system will settle to a post-disturbance steady-state that cannot equal the initial point, even though the parameters of the system are unchanged.

The WECC test system and default parameters of Appendix C illustrate this phenomenon. The pitch angle response of Figure 8 is repeated in Figure 11, where the time horizon has been extended to 30 seconds. Notice that the pitch angle evolves to a steady-state value of $0.33 \mathrm{deg}$, even though it was initialized at $0 \mathrm{deg}$.

Figure 12 shows the relationship between $T_{\omega}$ and $x_{p}$. These two states initially follow a straight line given by $(24)$, with the states reaching the extreme point $\left(T_{\omega}, x_{p}\right)=(0.847,0.56)$, before returning along the line to $(0.835,0.08)$. At that instant, the pitch angle $\theta$ encounters its limit, causing the integrator driving $x_{p}$ to block. The integrator remains blocked until $\theta$ enters a period where hysteresis is active. During that period, the $x_{p}$-integrator successively blocks and unblocks, giving rise to the staircase phenomenon apparent in the figure. At the end of that period, $\theta$ finally comes off its limit, the $x_{p}$-integrator is restored to normal operation, and the behaviour of $T_{\omega}$ and $x_{p}$ reverts to a straight-line locus given by an affine 


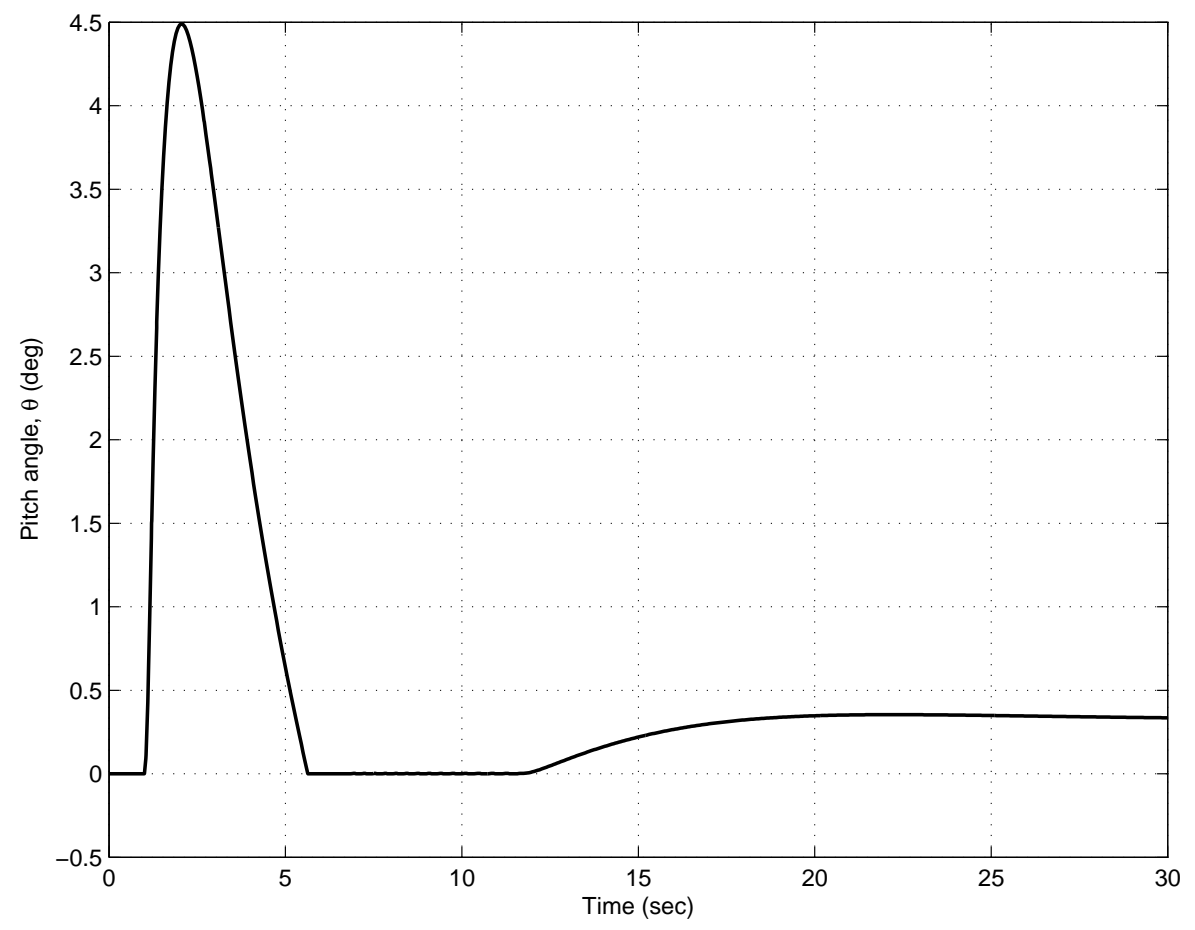

Figure 11: Large-disturbance response of pitch angle $\theta$.

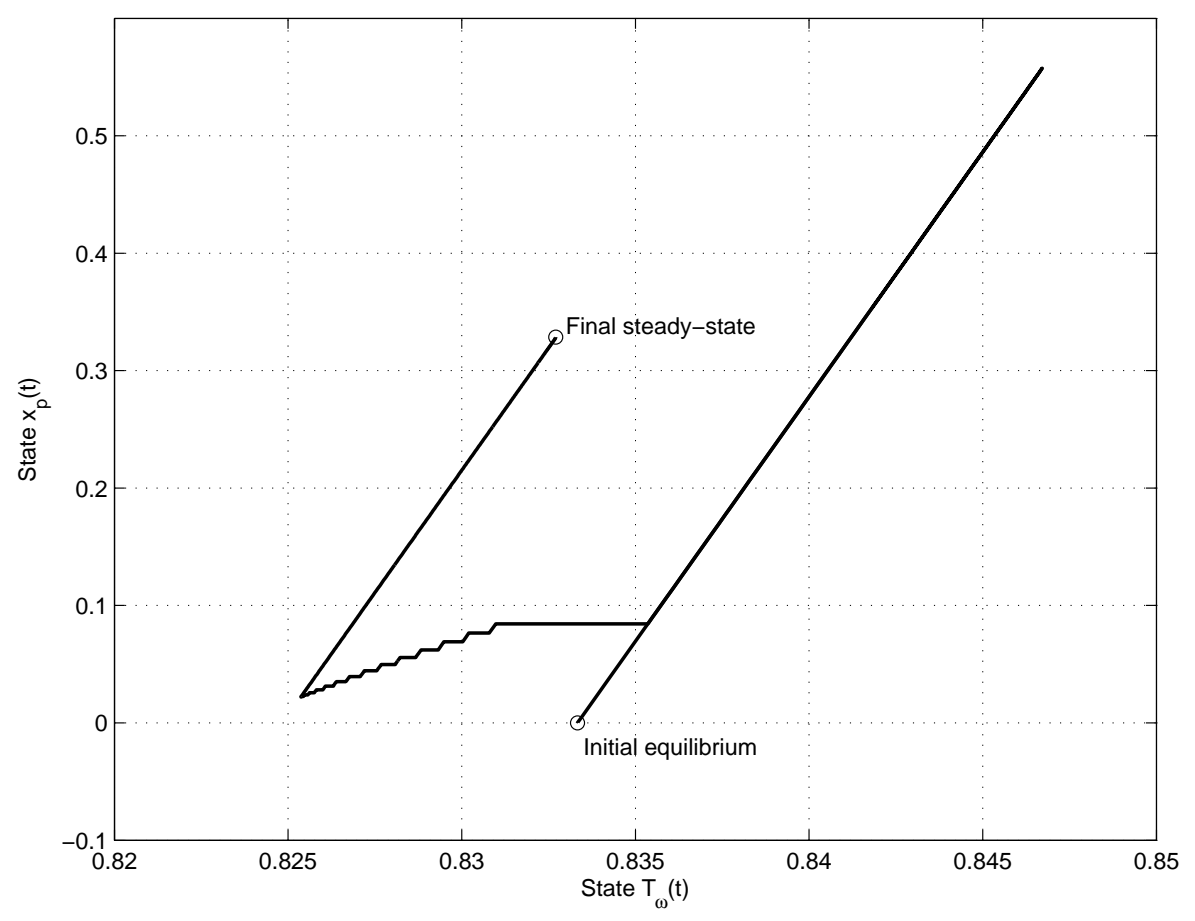

Figure 12: Relationship between $T_{\omega}(t)$ and $x_{p}(t)$. 
relationship like (24). The slope over that final section is the same as earlier, but the offset has changed. Hence the system converges to a steady-state that differs from the starting equilibrium point.

\section{Small disturbance analysis}

\subsection{Singularity}

Linearizing the WTG equations (1)-(11) about an equilibrium point, and eliminating the algebraic equations, yields the seventh-order linear model,

$$
\frac{d}{d t}\left[\begin{array}{c}
\Delta \omega_{\text {ref }} \\
\Delta T_{\omega} \\
\Delta P_{\text {ord }} \\
\Delta x_{p} \\
\Delta x_{c} \\
\Delta \theta \\
\Delta \omega
\end{array}\right]=\left[\begin{array}{ccccccc}
a_{11} & \cdot & a_{13} & \cdot & \cdot & \cdot & \cdot \\
a_{21} & \cdot & \cdot & \cdot & \cdot & \cdot & a_{27} \\
a_{31} & a_{32} & a_{33} & \cdot & \cdot & \cdot & a_{37} \\
a_{41} & \cdot & \cdot & \cdot & \cdot & \cdot & a_{47} \\
\cdot & \cdot & a_{53} & \cdot & \cdot & \cdot & \cdot \\
a_{61} & \cdot & a_{63} & a_{64} & a_{65} & a_{66} & a_{67} \\
\cdot & \cdot & a_{73} & \cdot & \cdot & a_{76} & \cdot
\end{array}\right]\left[\begin{array}{c}
\Delta \omega_{r e f} \\
\Delta T_{\omega} \\
\Delta P_{\text {ord }} \\
\Delta x_{p} \\
\Delta x_{c} \\
\Delta \theta \\
\Delta \omega
\end{array}\right]
$$

where the $a_{i j}$ refer to elements of the system $A$-matrix that are potentially non-zero, while all other elements are identically zero. The exact arrangement of the non-zero $a_{i j}$ is dependent upon the status of limits.

As in Section 4, it is assumed that none of the limits in the converter control model WT3E are enforced at steady-state, and that pitch angle $\theta$ dynamics are active. In the case of the pitch compensation $x_{c}$-integrator, its dynamics may be active at steady-state or the limiter may be enforced, as indicated in Figure 10. Both situations must be considered.

The initial value $\theta_{o}$ for the pitch angle also has an important influence on the linear model. When (11) is linearized with $\theta_{o}=0$, the term associated with the simplified aerodynamic model becomes zero. In that case, perturbations in the pitch angle $\theta$ have no influence on $P_{\text {mech }}$, and as a consequence $a_{76}=0$ in (25). On the other hand, when $\theta_{0} \neq 0$, the aerodynamic model contributes a non-zero term to (11), resulting in $a_{76} \neq 0$.

In considering the various cases identified above, it should be kept in mind that (2) and (5) are duplicate integrators. Because these integrators differ only by a scaling factor, the corresponding rows of the $A$-matrix, 2 and 4 respectively, are linearly dependent. Therefore $A$ must have at least one eigenvalue whose value is zero.

The two conditions for the $x_{c}$-integrator, together with the two possibilities for $\theta_{o}$, give four separate cases:

\subsection{1 $x_{c}$-integrator active, $\theta_{o} \neq 0$}

The condition $\theta_{o} \neq 0$ implies $a_{76} \neq 0$, so all the $a_{i j}$ shown in (25) are non-zero. The $A$-matrix has a single zero eigenvalue due to the linear dependence of rows 2 and 4, which correspond to the duplicate integrators. By inspection, columns 4 and 5 are linearly dependent, implying that the right eigenvector associated with the zero eigenvalue involves only $\Delta x_{p}$ and $\Delta x_{c}$. Substituting that zero-eigenvector ${ }^{4}$ into (25) gives a matrix-vector product that is zero. Hence, the linear system will be in steady-state at any point along the zero-eigenvector.

\footnotetext{
${ }^{4}$ For convenience, the right eigenvector associated with the zero eigenvalue will be referred to as the zeroeigenvector.
} 


\subsection{2 $x_{c}$-integrator active, $\theta_{o}=0$}

In this case $\theta_{o}=0$ so $a_{76}=0$. By inspection, rows 5 and 7 are linearly dependent, as well as rows 2 and 4 , implying the system now has two eigenvalues that are zero. This second zero-eigenvalue is a consequence of pitch angle $\Delta \theta$ being decoupled from mechanical power $\Delta P_{\text {mech }}$ when $\theta_{o}=0$ in the simplified aerodynamic model. With $a_{76}=0$, columns 4,5 and 6 are clearly linearly dependent. The two zero-eigenvectors in this case involve $\Delta x_{p}, \Delta x_{c}$ and $\Delta \theta$. The linear system will be in steady-state at any point on the plane spanned by the two zero-eigenvectors.

\subsection{3 $x_{c}$-integrator blocked, $\theta_{o} \neq 0$}

Blocking the $x_{c}$-integrator implies $\Delta x_{c} \equiv 0$. Accordingly, the fifth row and column of the $A$-matrix should be removed, reducing the linearized system to six dynamic states. The linear dependence of rows 2 and 4 is unaffected by this reduction, so one of the eigenvalues remains zero. Because $a_{76} \neq 0$, no simple pairing of columns produces linear dependence. In fact, linear dependence involves all the columns of the reduced $A$-matrix. Accordingly, the zero-eigenvector includes a contribution from all six states of the reduced model.

\subsection{4 $x_{c}$-integrator blocked, $\theta_{o}=0$}

This case also has $\Delta x_{c} \equiv 0$, so the $A$-matrix is again reduced. Because $\theta_{o}=0$, the element $a_{76}=0$, and by inspection the columns corresponding to $\Delta x_{p}$ and $\Delta \theta$ are linearly dependent. The zero-eigenvector therefore involves only $\Delta x_{p}$ and $\Delta \theta$.

\subsection{Eliminating the zero eigenvalue}

It is possible to eliminate the zero eigenvalue caused by integrator duplication by exploiting the explicit coupling between integrator states $T_{\omega}$ and $x_{p}$ given by (24). Linearizing that affine relationship gives

$$
\Delta x_{p}(t)=\frac{K_{i p}}{K_{i t r q}} \Delta T_{w}(t)
$$

In the linearized model (25), removing the row corresponding to $\Delta x_{p}$, and replacing all occurrences of $\Delta x_{p}$ by (26), reduces the system dimension by one. It can be shown that the remaining eigenvalues are exactly the same as the original non-zero eigenvalues. If those original non-zero eigenvalues all have negative real parts, as is generically the case, the system will be exponentially stable.

Even though the linear model has a continuum of equilibria defined by the zero-eigenvector, perturbations that satisfy (26) will induce behaviour that returns to the original equilibrium point. On the other hand, perturbations that do not satisfy (26) will result in convergence to points on the zero-eigenvector that generically differ from the original point.

\subsection{Linearizing at limits}

Linearization about an equilibrium point requires the dynamical system to be smooth in a neighbourhood of that point. With hybrid dynamical systems, such as the WTG model, equilibria may coincide with conditions that induce switching. In such cases, it is impossible 
to find a neighbourhood of the equilibrium point where the dynamical system is smooth. Linearization is therefore not well defined.

Consider the pitch compensation $x_{c}$-integrator, whose equilibrium characteristic is provided in Figure 10. Linearizing about an equilibrium point anywhere on the vertical or horizontal sections of the characteristic, away from the transition point at the origin, is well defined and discussed in Section 5.1. At the origin, however, switching will occur as perturbations in $x_{c}$ transition from positive to zero. To establish a linear model at this point, switching must be disabled. The $x_{c}$-limit may be ignored, so the origin behaves like a point on the vertical characteristic, or it may be enforced, in which case the origin will act like a point on the horizontal characteristic. It is important to note that the two cases will result in different linear models, and hence eigen-structures that differ. Neither is strictly correct, and results must be interpreted with great care.

A similar discussion applies for the pitch angle $\theta$ dynamics. As mentioned in Section 4.1, it is common for $\theta$ to be initialized on its lower limit $P I_{\min }$. A choice must be made whether to treat the integrator as active or blocked. The analysis of Section 5.1 was based on the assumption that the integrator was active.

The ill-defined nature of linearization at a switching point is particularly important for analysis packages that use finite differences to generate approximate derivatives. Care must be taken to ensure that differences are calculated using perturbations that are physically meaningful. Otherwise the linear model may be quite inaccurate.

\section{Conclusions}

The dynamic behaviour of type-3 WTGs is governed by interactions between a wound-rotor induction machine and a back-to-back inverter. The inverter response time is much faster than the time constants of the induction machine, allowing the inverter to respond rapidly to the electromechanical dynamics of the WTG. Consequently, the dynamic characteristics of a type-3 WTG that are important from the grid perspective are dominated by the response of controllers that regulate active power, pitch angle and terminal voltage. These controllers involve interactions between continuous dynamics of state variables and discrete events that occur when limits are encountered. WTGs may therefore be classed as hybrid dynamical systems.

Non-windup limits within the WECC type-3 WTG model have been structured so that various integrators are blocked when a limit is encountered. The interactions inherent in this model structure can be quite complex, and may lead to a form of trajectory deadlock known as chattering Zeno. Deadlock precludes continuation of the trajectory in the normal sense, so numerical simulation techniques that accurately capture hybrid system dynamics cannot proceed beyond such a point. Filippov solution concepts are required for continuing the trajectory. It has been shown that deadlock can be eliminated by incorporating hysteresis into the switching of non-windup limits.

The converter controller and the pitch controller of the WECC type-3 WTG model both include an integrator that is driven by the same frequency error signal. This integrator duplication results in an under-determined description of steady-state conditions, allowing the existence of a continuum of equilibria. As a consequence, power systems that incorporate WTGs may converge to a post-disturbance steady-state that does not match initial conditions, even though the parameter set has not changed. Furthermore, the duplicate integrators result 
in linearly dependent rows in the linearized WTG model, so the system is singular. Small disturbance analysis of power systems with WTGs will yield at least one zero-eigenvalue for every WTG.

\section{A Switched equations for WT3E}

In the following set of switched equations, (27) implements the rate limits associated with the $P_{\text {ord }}$ block, establishing the rate-limited signal $P_{\text {ord,rtlm }}$ that drives $P_{\text {ord }}$ in (3). Equations (28) and (29) implement the $P_{\max }$ and $P_{\min }$ non-windup limits respectively. Consider the $P_{\max }$ limit described by (28). While $P_{\text {ord }} \leq P_{\max }$, the trigger variable $y_{m x} \geq 0$, so the switch $y_{m x, s w}=1$. This ensures the integrator (3) is not blocked. On the other hand, if system conditions sought to drive $P_{\text {ord }}$ beyond $P_{\max }$, then $y_{m x}$ would immediately change sign, triggering $y_{m x, s w}$ to switch to 0 . That would force the right hand side of integrator (3) to zero, ensuring $P_{\text {ord }}$ was frozen at its $P_{\max }$ limit. While $P_{\text {ord,rtlm }}$ remained positive, seeking to drive $P_{\text {ord }}$ harder onto the limit, the trigger variable $y_{m x}<0$. As soon as $P_{\text {ord,rtlm }}$ changed sign though, allowing $P_{\text {ord }}$ to come off its limit, $y_{m x}$ would also change sign, and $y_{m x, s w}$ would switch to 1 , thus re-enabling the integrator (3). Similar logic applies in (29) for the $P_{\min }$ limit.

Equation (30) enforces the rules specified in (i) and (ii) of Section 2.1 that link the blocking of the PI-integrator with the $P_{\text {ord }}$ limit conditions. It does so through the use of switch variable $y_{\text {freeze }}$ that is used to turn on/off the integrator (2).

$$
\begin{aligned}
& \begin{array}{l}
\text { if } P_{\text {ord }, \text { rate }}>d P_{\max } / d t \\
\quad P_{\text {ord }, \text { rtlm }}=d P_{\max } / d t \\
\text { elseif } P_{\text {ord,rate }}<-d P_{\max } / d t \\
\qquad P_{\text {ord,rtlm }}=-d P_{\max } / d t \\
\text { else } \\
\quad P_{\text {ord }, \text { rtlm }}=P_{\text {ord,rate }} \\
\text { endif }
\end{array} \\
& \text { if } y_{m x} \geq 0 \\
& y_{m x}=P_{\text {max }}-P_{\text {ord }} \\
& y_{m x, s w}=1 \\
& \text { else } \\
& y_{m x}=-P_{\text {ord }, r t l m} \\
& y_{m x, s w}=0 \\
& \text { endif }
\end{aligned}
$$




$$
\begin{aligned}
& \text { if } \begin{array}{l}
y_{m n} \geq 0 \\
\quad y_{m n}=P_{\text {ord }}-P_{\text {min }}
\end{array} \\
& \quad y_{m n, s w}=1 \\
& \text { else } \\
& \quad y_{m n}=P_{\text {ord,rtlm }} \\
& \quad y_{m n, s w}=0 \\
& \text { endif } \\
& \text { if } y_{m x, \text { sw }}=0 \text { and } \omega-\omega_{\text {ref }}>0 \\
& \quad y_{\text {freeze }}=0 \\
& \text { elseif } y_{m n, s w}=0 \text { and } \omega-\omega_{r e f}<0 \\
& \quad y_{\text {freeze }}=0 \\
& \text { else } \\
& \quad y_{\text {freeze }}=1 \\
& \text { endif }
\end{aligned}
$$

\section{B Switched equations for WT3P}

In the following set of switched equations, (31) implements the rate limit associated with the $\theta$ block, establishing the rate-limited signal $\theta_{\text {rtlm }}$ that drives $\theta$ in (7). Equations (32) and (33) implement the $P I_{\max }$ and $P I_{\min }$ non-windup limits respectively, while (34) models the lower non-windup limit on the pitch compensation integrator. Equations (35) and (36) enforce the rules that link the blocking of the pitch-control and pitch-compensation integrators to the $P I_{\max } / P I_{\min }$ non-windup limits.

$$
\begin{aligned}
& \left.\begin{array}{l}
\text { if } \theta_{\text {rate }}>P I_{\text {rate }} \\
\quad \theta_{\text {rtlm }}=P I_{\text {rate }} \\
\text { else } \\
\qquad \theta_{\text {rtlm }}=\theta_{\text {rate }} \\
\text { endif }
\end{array}\right\} \\
& \text { if } y_{m x} \geq 0 \\
& y_{\text {mx }}=P I_{\text {max }}-\theta \\
& y_{m x, s w}=1 \\
& \text { else } \\
& y_{m x}=-\theta_{r t l m} \\
& y_{m x, s w}=0 \\
& \text { endif }
\end{aligned}
$$




$$
\begin{aligned}
& \text { if } y_{m n} \geq 0 \\
& y_{m n}=\theta-P I_{\min } \\
& y_{m n, s w}=1 \\
& \text { else } \\
& y_{m n}=\theta_{r t l m} \\
& y_{m n, s w}=0 \\
& \text { endif } \\
& \text { if } y_{x c} \geq 0 \\
& y_{x c}=x_{c}-x_{c, l i m} \\
& y_{s w}=1 \\
& y_{x c}=P_{\text {ord }}-P_{\text {set }} \\
& y_{s w}=0 \\
& y_{f r, 1}=0 \\
& \text { elseif } y_{m n, s w}=0 \text { and } \omega-\omega_{\text {ref }}<0 \\
& y_{f r, 1}=0 \\
& y_{f r, 1}=1 \\
& y_{f r, 2}=0 \\
& y_{f r, 2}=0 \\
& y_{f r, 2}=1 \\
& \}
\end{aligned}
$$

\section{WECC default parameter values}

The test system is given in Figure 13. Parameter values are given in Tables 1 to 7. The wind generator output is $100 \mathrm{MW}=1.0 \mathrm{pu}$. A 10 MVAr capacitor is connected at bus 3 , but no shunt is connected at bus 5 .

A three-phase fault is applied at bus 2 at 1 sec. It is cleared at $1.15 \mathrm{sec}$ by disconnecting one of the $230 \mathrm{kV}$ transmission lines. 


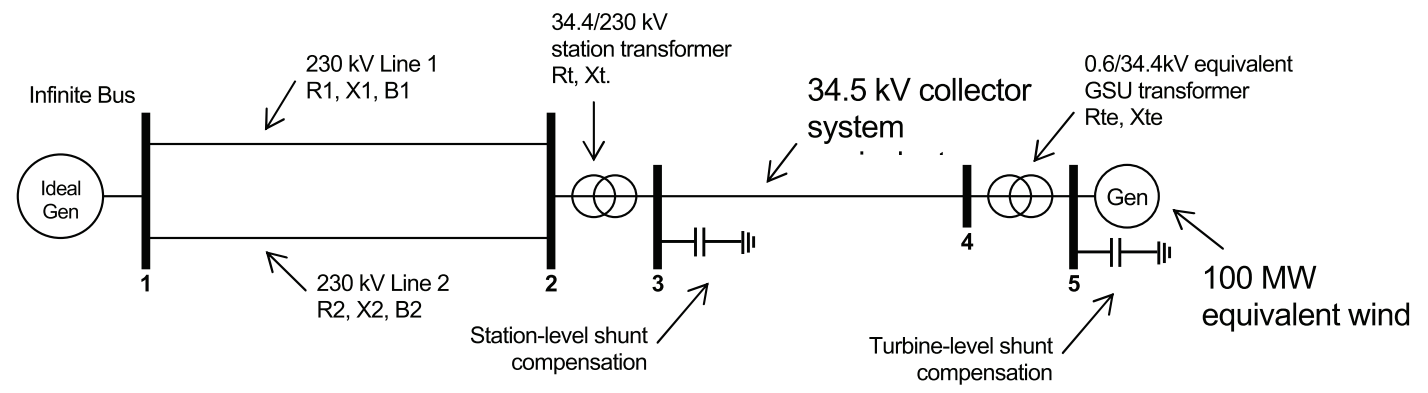

Figure 13: Standard test system for evaluating WTG dynamic performance.

Table 1: Impedance values for the test system of Figure 13.

\begin{tabular}{|c|c|c|c|c|}
\hline$R_{1}=R_{2}$ & $X_{1}=X_{2}$ & $B_{1}=B_{2}$ & $R_{t}$ & $X_{t}$ \\
\hline 0.025 & 0.250 & 0.05 & 0.0 & 0.1 \\
\hline \hline$R_{e}$ & $X_{e}$ & $B_{e}$ & $R_{t e}$ & $X_{t e}$ \\
\hline 0.015 & 0.025 & 0.01 & 0.0 & 0.05 \\
\hline
\end{tabular}

Table 2: Parameter values for WT3G.

\begin{tabular}{|c|c|c|c|}
\hline$X_{e q}$ & $K_{\text {pll }}$ & $K_{\text {ipll }}$ & $P_{\text {llmax }}$ \\
\hline \hline 0.8 & 30 & 1 & 0.1 \\
\hline
\end{tabular}

Table 3: Parameter values for WT3E reactive power control.

\begin{tabular}{|c|c|c|c|c|c|}
\hline varflg & vltflg & $V_{\text {ref }}$ & $K_{q i}$ & $V_{\max }$ & $V_{\min }$ \\
\hline \hline 0 & 0 & 1 & 0.1 & 1.1 & 0.9 \\
\hline
\end{tabular}

Table 4: Parameter values for WT3E active power (torque) control.

\begin{tabular}{|c|c|c|c|c|c|c|c|}
\hline$K_{p t r q}$ & $K_{i t r q}$ & $T_{p c}$ & $P_{\min }$ & $P_{\max }$ & $I_{p \max }$ & $d P_{\max } / d t$ & $T_{s p}$ \\
\hline \hline 3 & 0.6 & 0.05 & 0.04 & 1.12 & 1.1 & 0.45 & 5 \\
\hline
\end{tabular}

Table 5: Parameter values for speed-power curve $f\left(P_{g e n}\right)$.

\begin{tabular}{|c|c|c|c|c|c|}
\hline$\omega_{p \min }$ & $\omega_{p 20}$ & $\omega_{p 40}$ & $\omega_{p 60}$ & $P_{\omega p 100}$ & $\omega_{p 100}$ \\
\hline \hline 0.69 & 0.78 & 0.98 & 1.12 & 0.74 & 1.2 \\
\hline
\end{tabular}

Table 6: Parameter values for WT3T single-shaft model.

\begin{tabular}{|c|c|c|c|c|}
\hline$K_{\text {aero }}$ & $\theta_{0}$ & $P_{m 0}$ & $H$ & $D$ \\
\hline \hline 0.007 & 0 & 1 & 4.94 & 0 \\
\hline
\end{tabular}

Table 7: Parameter values for WT3P.

\begin{tabular}{|c|c|c|c|c|c|c|c|c|}
\hline$K_{p p}$ & $K_{i p}$ & $K_{p c}$ & $K_{i c}$ & $T_{P I}$ & $P I_{\max }$ & $P I_{\min }$ & $P I_{\text {rate }}$ & $P_{\text {set }}$ \\
\hline \hline 150 & 25 & 3 & 30 & 0.3 & 27 & 0 & 10 & 1 \\
\hline
\end{tabular}




\section{References}

[1] T. Ackermann (Editor), Wind Power in Power Systems. England: John Wiley and Sons, 2005.

[2] E. Muljadi, C. Butterfield, B. Parsons, and A. Ellis, "Effect of variable speed wind turbine generator on stability of a weak grid," IEEE Transactions on Energy Conversion, vol. 22, no. 1, pp. 29-36, March 2007.

[3] D. Gautam, V. Vittal, and T. Harbour, "Impact of increased penetration of DFIG-based wind turbine generators on transient and small signal stability of power systems," IEEE Transactions on Power Systems, vol. 24, no. 3, pp. 1426-1434, August 2009.

[4] M. Nunes, J. Lopes, H. Zurn, U. Bezerra, and R. Almeida, "Influence of the variablespeed wind generators in transient stability margin of the conventional generators integrated in electrical grids," IEEE Transactions on Energy Conversion, vol. 19, no. 4, pp. 692-701, December 2004.

[5] N. Ullah and T. Thiringer, "Effect of operational modes of a wind farm on the transient stability of nearby generators and on power oscillations: a Nordic grid study," Wind Energy, vol. 11, pp. 63-73, September 2007.

[6] D. Kosterev and D. Davies, "System model validation studies in WECC," in Proceedings of the IEEE PES General Meeting, Minneapolis, MN, July 2010.

[7] I. Hiskens and J. Alseddiqui, "Sensitivity, approximation and uncertainty in power system dynamic simulation," IEEE Transactions on Power Systems, vol. 21, no. 4, pp. 18081820, November 2006.

[8] J. Slootweg, S. de Haan, H. Polinder, and W. Kling, "General model for representing variable speed wind turbines in power system dynamics simulations," IEEE Transactions on Power Systems, vol. 18, no. 1, pp. 144-151, February 2003.

[9] J. Ekanayake, L. Holdsworth, X.-G. Wu, and N. Jenkins, "Dynamic modeling of doubly fed induction generator wind turbines," IEEE Transactions on Power Systems, vol. 18, no. 2, pp. 803-809, May 2003.

[10] Y. Lei, A. Mullane, G. Lightbody, and R. Yacamini, "Modeling of the wind turbine with a doubly fed induction generator for grid integration studies," IEEE Transactions on Energy Conversion, vol. 21, no. 1, pp. 257-264, March 2006.

[11] M. Kayikçi and J. Milanović, "Assessing transient response of DFIG-based wind plants The influence of model simplifications and parameters," IEEE Transactions on Power Systems, vol. 23, no. 2, pp. 545-554, May 2008.

[12] J. Rose and I. Hiskens, "Estimating wind turbine parameters and quantifying their effects on dynamic behavior," in Proceedings of the IEEE PES General Meeting, Pittsburgh, PA, July 2008.

[13] K. Clark, N. Miller, and J. Sanchez-Gasca, "Modeling of GE wind turbine-generators for grid studies," Version 4.4, GE Energy, September 2009. 
[14] E. Muljadi, C. Butterfield, J. Conto, and K. Donoho, "ERCOT's dynamic model of wind turbine generators," in Proceedings of WindPower 2005, Denver, CO, May 2005.

[15] "Generic type-3 wind turbine-generator model for grid studies," Version 1.1, WECC Wind Generator Modeling Group, September 2006.

[16] Z. Lubosny, Wind Turbine Operation in Electric Power Systems. Springer, 2003.

[17] A. van der Schaft and H. Schumacher, An Introduction to Hybrid Dynamical Systems. London: Springer-Verlag, 2000.

[18] D. Liberzon, Switching in Systems and Control. Boston: Birkhauser, 2003.

[19] I. Hiskens, "Power system modeling for inverse problems," IEEE Transactions on Circuits and Systems I: Regular Papers, vol. 51, no. 3, pp. 539-551, March 2004.

[20] IEEE Std 421.5-2005, IEEE Recommended Practice for Excitation System Models for Power System Stability Studies. New York: Institute of Electrical and Electronics Engineers, Inc., 2005.

[21] A. Abate, A. D'Innocenzo, M. Di Benedetto, and S. Sastry, "Understanding deadlock and livelock behaviors in hybrid control systems," Nonlinear Analysis: Hybrid Systems, vol. 3, no. 2, pp. 150-162, May 2009.

[22] I. Hiskens and P. Sokolowski, "Systematic modeling and symbolically assisted simulation of power systems," IEEE Transactions on Power Systems, vol. 16, no. 2, pp. 229-234, May 2001.

[23] H. Khalil, Nonlinear Systems, 3rd ed. Upper Saddle River, NJ: Prentice Hall, 2001.

[24] I. Hiskens and D. Hill, "Energy functions, transient stability and voltage behaviour in power systems with nonlinear loads," IEEE Transactions on Power Systems, vol. 4, no. 4, pp. 1525-1533, November 1989.

[25] V. Venkatasubramanian, H. Schättler, and J. Zaborszky, "Dynamics of large constrained nonlinear systems - a taxonomy theory," Proceedings of the IEEE, vol. 83, no. 11, pp. 1530-1561, November 1995.

[26] A. Filippov, Differential Equations with Discontinuous Righthand Sides. The Netherlands: Kluwer Academic Publishers, 1988.

[27] V. Utkin, J. Guldner, and J. Shi, Sliding Mode Control in Electro-Mechanical Systems, 2nd ed. CRC Press, 2009.

[28] I. Hiskens, "Trajectory deadlock in power system models," in Proceedings of the IEEE International Symposium on Circuits and Systems, Rio de Janeiro, Brazil, May 2011, to appear.

[29] R. Cottle, J.-S. Pang, and R. Stone, The Linear Complementarity Problem. Boston, MA: Academic Press, 1992.

[30] A. van der Schaft and J. Schumacher, "Complementarity modeling of hybrid systems," IEEE Transactions on Automatic Control, vol. 43, no. 4, pp. 483-490, April 1998. 